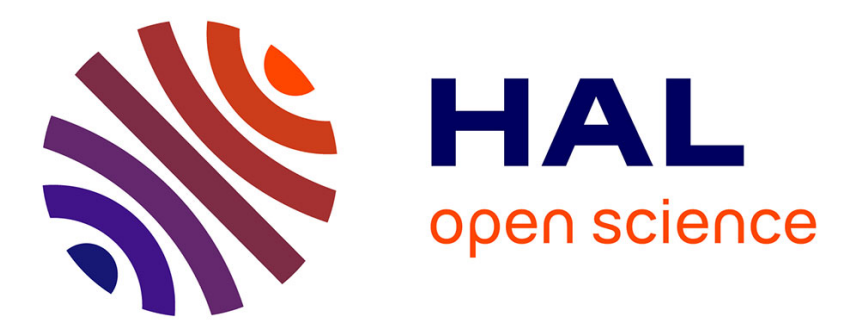

\title{
Chérie et Le Docteur Pascal : filiation et transmission des héritages \\ Dominique Pety
}

\section{To cite this version:}

Dominique Pety. Chérie et Le Docteur Pascal : filiation et transmission des héritages. Cahiers Edmond et Jules de Goncourt, 2013, Le Roman de la jeune fille, 1 (20), pp.105-123. 10.3406/cejdg.2013.1148 . hal-02466632

\section{HAL Id: hal-02466632 \\ https://hal.univ-smb.fr/hal-02466632}

Submitted on 4 Feb 2020

HAL is a multi-disciplinary open access archive for the deposit and dissemination of scientific research documents, whether they are published or not. The documents may come from teaching and research institutions in France or abroad, or from public or private research centers.
L'archive ouverte pluridisciplinaire HAL, est destinée au dépôt et à la diffusion de documents scientifiques de niveau recherche, publiés ou non, émanant des établissements d'enseignement et de recherche français ou étrangers, des laboratoires publics ou privés. 


\section{Chérie et Le Docteur Pascal : filiation et transmission des héritages}

C'est dans La Joie de vivre qu'Edmond de Goncourt avait cru voir, fin 1883, son dernier roman Chérie plagié par son rival de toujours ${ }^{1}$. Or c'est un autre roman de Zola, publié dix ans plus tard, qui doit probablement beaucoup à Chérie, en vertu d'une communauté structurelle et thématique qui met magistralement en valeur les orientations esthétiques et idéologiques radicalement opposées des deux romanciers en cette fin du XIX ${ }^{e}$ siècle : comme si, pour le dire rapidement, Zola avait voulu réécrire en partie Chérie pour lui donner une autre fin, et conclure son cycle des Rougon-Macquart dans un jeu d'échos avec un roman qu'Edmond de Goncourt avait pour sa part programmé comme le dernier des siens.

Ce sont ces configurations similaires que nous essaierons de mettre en lumière, pour montrer comment, derrière ce qui s'apparente au roman de formation d'une jeune fille, sur fond d'un décor qui symbolise tout un héritage culturel, Goncourt et Zola apportent chacun leur propre réponse à une question qui taraude la deuxième moitié du siècle, à savoir le poids de l'ancien et la façon dont il oblitère, ou non, les promesses de renouveau et la capacité de l'avenir à s'inventer ou se ré-inventer'.

\section{La jeune fille et le vieil homme, ou Pygmalion et Galatée}

A priori, rien de commun entre les deux romans, dont la parenté n'a jamais été suggérée par la critique. Leur titre même semble mettre l'accent sur deux personnages que tout oppose. Dans un cas, une jeune Parisienne qui meurt hystérique et anémiée à dix-neuf ans, dans l'autre un médecin de province qui succombe à une maladie de poitrine à cinquante-neuf ans.

1. La publication de La Joie de vivre en feuilleton du 29 novembre 1883 au 3 février 1884 précède de peu la publication de Chérie dans le même journal. Edmond de Goncourt, qui avait fait des lectures publiques de plusieurs extraits de son roman, se croit alors plagié, notamment dans le chapitre II de Zola qui évoque la puberté de Pauline. Zola dément formellement dans une lettre à Edmond du 14 décembre. (Voir notamment E. et J. de Goncourt, Journal, 2 novembre et 15 décembre 1883).

2. Un article de Jean-Louis Cabanès sur les représentations du vivant a récemment mis en valeur, au tournant des $\mathrm{XIX}^{\mathrm{e}}$ et $\mathrm{XX}^{\mathrm{c}}$ siècles, un discours conjoint de la philosophie et de la biologie « sur la continuité de la vie, sur la poussée du passé dans le présent ", et la place charnière qu'occupe $L e$ Docteur Pascal (1893) dans ce dispositif, en ce qu'il figure "à la fois une fin et un commencement " (J.-L. Cabanès, "Les valeurs du vivant au tournant des $\mathrm{XIX}^{\mathrm{e}}$ et $\mathrm{XX}^{\mathrm{e}}$ siècles », Romantisme, "Le Vivant ", n $154,2011-4$, p. 105-122, citation p. 111). 
Cependant, au-delà de la différence marquée par les titres, les deux romans mettent au premier plan un couple de structure analogue, une jeune fille et un vieil homme, lequel a recueilli la première dès l'enfance, dans un contexte où la filiation est d'emblée posée comme problématique. Chez Goncourt, il s'agit d'un grand-père entièrement dévoué à sa petite-fille orpheline, et chez Zola, d'un oncle qui a accepté d'élever sa nièce dont le père s'est remarié. Ce qui frappe cependant, à la lecture de ces deux romans, c'est le destin radicalement opposé qui s'offre à ces deux jeunes femmes. À la première, Chérie, qu'on a vu longuement grandir (cette croissance est l'objet même du roman), est refusée la possibilité de vivre (et de quitter la sphère de l'enfance et de la sujétion) ${ }^{1}$, et c'est son vieux grand-père qui lui survit. Quant à la seconde, Clotilde, dont l'affection filiale pour son oncle évolue vers une relation amoureuse partagée, elle va au contraire incarner la vie qui se prolonge dans l'enfant qu'elle met au monde, tandis que meurt l'oncle, père de l'enfant. Cette différence radicale dans le destin du personnage féminin, sur fond d'une analogie structurelle dans la répartition des deux rôles principaux, dans deux romans que moins de dix ans séparent, invite à s'interroger sur l'enjeu esthétique et philosophique de ces deux conclusions, l'une fermée et que l'on peut inscrire, à la lecture du texte préfaciel, dans un contexte intellectuel et culturel également arrivé à son terme, l'autre au contraire symboliquement ouverte sur un futur à venir, avec l'enfant au bras dressé « comme un drapeau d'appel à la vie ${ }^{2}$ ".

Un imaginaire archä̈que, qu'illustre bien le mythe de Pygmalion et Galatée, informe sans doute les deux romans, non seulement au niveau de l'intrigue (le devenir d'une jeune fille élevée avec soin et presque façonnée par un personnage qui joue symboliquement le rôle du père), mais aussi parce que ce personnage masculin est, à bien des égards, comme on le verra plus loin, une transposition du romancier.

Chez Goncourt, le grand-père Haudancourt est certes moins le créateur que l'adorateur de la petite fille qu'il voit grandir ${ }^{4}$. Mais c'est bien son regard qui nous invite à percevoir en l'enfant une ouvre d'art en puissance :

Chérie commence à devenir une de ces miniatures de femmes minuscules, telles qu'on les rencontre dans les amusantes imageries en couleur des almanachs dessinés par Kate Greenaway, mais moins raide, mais d'une ingénuité physique plus capricieuse de lignes, plus contournée, plus parmegianesque.

1. Je n'évoque pas ici le discours médical de l'époque auquel Edmond de Goncourt se conforme en montrant son héroïne succombant à l'hystérie d'une sexualité contrariée par les lois sociales. 2. Le Docteur Pascal, éd. Jean-Louis Cabanès, Paris, LGF (Livre de poche Classiques), 2004, p. 429.

3. Anne Geisler a analysé la réactivation de ce mythe au XIX siècle (Le Mythe de Pygmalion au XIX siècle. Pour une approche de la coalescence des mythes, Paris, Champion, 1999).

4. Chérie, éd. Jean-Louis Cabanès et Philippe Hamon, Jaignes, La Chasse au Snark (Collection de la Société des études romantiques), 2002. Voir notamment chap. VI, p. 83. Je renvoie aussi à mon édition, à paraître aux éditions Classiques Garnier. 
Le vieux maréchal, à la fois surpris et charmé de la métamorphose de sa petite-fille, sans pouvoir en détacher les yeux, la regarde profilée en sa silhouette rigide, les coudes rapprochés du corps dans un resserrement qu'on dirait frileux, un pied relevé, et où, dans le bout de sa bottine recroquevillée qu'elle écrase contre la terre, les doigts grandissants ont une vague ressemblance avec les familles des pieds des statues changées en arbres; il la regarde, son mince corps serré dans une robe étroite moulant sa délicate maigreur, pendant qu'elle court, les cheveux envolés au dos, avec la projection en avant d'une petite Atalante ; il la regarde encore épelant son premier conte de fée, assise sur un haut banc de jardin, les jambes ballantes, une main soutenant le coude du bras qui tient le livre, dans une immobilité gracieusement remuante ${ }^{1}$.

Ce que le grand-père crée à proprement parler, et qui témoigne aussi de cette admiration qui confine à l'adoration, c'est une rose qu'il baptise du nom de sa petite-fille, geste par lequel il espère lui conférer une sorte "d'immortalité " ». C'est là une nouvelle préfiguration de la supériorité quasi divine peu à peu attribuée à la jeune fille, œuvre d'art animée, telle la statue Galatée à laquelle Aphrodite accorde la vie.

Chez Zola, si Pascal est un Pygmalion, c'est sous les traits du savant qui expérimente, et qui tente de façonner un jeune être selon sa propre pensée. Dès le premier chapitre apparaît le regret de Pascal de n'avoir pas été plus radical dans sa démarche, lorsqu'il voit Clotilde se rebeller et tenter de détruire ses dossiers :

Toi que j'ai faite, toi qui es mon élève, mon amie, mon autre pensée, à qui j'ai donné un peu de mon cœur et de mon cerveau! Ah! oui, j’aurais dû te garder tout entière pour moi, ne pas me laisser prendre le meilleur de toi-même par ton bête de Bon Dieu ${ }^{3}$ !

Ce leitmotiv revient au dernier chapitre, mais cette fois il est formulé par Clotilde elle-même dans un mouvement d'acceptation et de reconnaissance :

Et sa pensée, alors, sauta à la gratitude profonde qu'elle gardait à Pascal de l'avoir faite ce qu'elle était. Jadis, lorsque, toute petite, l'enlevant à un milieu exécrable, il l'avait prise avec lui, il avait sûrement cédé à son bon cœur, mais sans doute aussi était-il désireux de tenter sur elle l'expérience de savoir comment elle pousserait dans un milieu autre, tout de vérité et de tendresse. [...] Elle lui devait certainement le meilleur de son être, elle devinait la fantasque et la violente qu'elle aurait pu devenir, tandis qu'il ne lui avait donné que de la passion et du courage ${ }^{4}$.

Au delà de ce formatage intellectuel et moral, Clotilde est également considérée ponctuellement comme une ouvre d'art, mais par le biais d'une référence un peu convenue, et qui n'est pas ici explicitement rapportée au regard de Pascal :

1. Ibid., chap. XIII, p. 101.

2. Ibid., chap. XXIX, p. 132.

3. Le Docteur Pascal, op. cit., chap. I, p. 71.

4. Ibid., chap. XIV, p. 495. 
Dans sa longue blouse noire, elle était très grande, la taille mince, la gorge menue, le corps souple, de cette souplesse allongée des divines figures de la Renaissance. Malgré ses vingt-cinq ans, elle restait enfantine et en paraissait à peine dix-huit.

En revanche, c'est bien Pascal qui la pare comme une statue lorsqu'il devient son amant :

Elle était comme une idole, le dos contre l'oreiller, assise sur son séant, chargée d'or, avec un bandeau d'or dans les cheveux, de l'or à ses bras nus, de l'or à sa gorge nue, toute nue et divine, ruisselante d'or et de pierreries ${ }^{2}$.

Il a fait d'elle une femme dans la relation amoureuse, et il fait d'elle une déesse dans la vénération qu'il lui porte. C'est qu'elle est à son tour investie d'un pouvoir, celui de le faire renaittre :

Clotilde était le renouveau qui arrivait à Pascal sur le tard, au déclin de l'âge. Elle lui apportait du soleil et des fleurs, plein sa robe d'amante; et, cette jeunesse, elle la lui donnait après les trente années de son dur travail, lorsqu'il était las déjà, et pâlissant, d'être descendu dans l'épouvante des plaies humaines. Il renaissait sous ses grands yeux clairs, au souffle pur de son haleine ${ }^{3}$.

Si Clotilde conquiert ainsi une sorte de supériorité sur Pascal qui loue la beauté de sa jeunesse, elle n'en devient jamais dominatrice; en outre, dans la relation de savoir qui la lie à Pascal, si elle est parfois dans la posture de l'élève rebelle, elle ne met jamais en cause la précellence de celui qu'elle ne cesse de reconnaître pour maître. À Pascal le savoir théorique sur le vivant, et la capacité de modeler intellectuellement un être à son image, à Clotilde la beauté physique et la capacité effective de donner la vie ou de rendre à la vie dans la relation amoureuse.

Une réciprocité dans le couple s'amorce donc chez Zola, alors qu'elle se manifeste rarement dans Chérie : le grand-père va rapidement évoluer vers la posture du spectateur totalement impuissant, non seulement parce que maint chapitre insiste sur les caprices de l'enfant, mais surtout parce que Chérie est un être androgyne, qui incarne à la fois la créature et le créateur ${ }^{4}$. Par sa "science" de la parure, elle fabrique elle-même sa propre beauté. E. de Goncourt évoque ainsi la vie de la jeune fille qui a commencé à fréquenter les bals de la haute société :

1. Ibid., chap. 1, p. 43. Une référence explicite à Jean Goujon apparait dans le Dossier préparatoire, comme l'indique J.-L. Cabanès.

2. Ibid., chap. VIII, p. 286.

3. Ibid., chap. VIII, p. 276.

4. Voir mon article "Le décoratif, emblème du malaise fin-de-siècle dans Chérie", Cahiers

Edmond et Jules de Goncourt, $\mathrm{n}^{\circ}$ 16, 2009, p. 73-84. 
[...] son existence toute donnée au façonnement de son être en une figuration d'élégance, et de l'élégance portée au dernier raffinement, dans l'élaboration coquette de cette gloire, pour ainsi dire, apportée par la toilette au corps et au visage de la femme, parmi des aspirations féminines à chercher, dans les fantaisies de la mise et de la parure, une jolie et radieuse surhumanité $[\ldots]$

Edmond de Goncourt insiste ainsi sur la solitude et l'autosuffisance de Chérie, qui ne dépend plus du regard ou de l'action d'autrui pour exister en tant qu'œuvre d'art :

Chérie demeurait en sa chambre, en des adorations paresseuses de sa personne, dépensant de la coquetterie pour elle toute seule, et dérangée désagréablement par une visite ${ }^{2}[\ldots]$

Cette absence de relation à l'autre, en une perfection stérile qui masque mal un clivage (la violence que le corps naturel exerce sur le corps paré), préfigure sans doute la mort. Ainsi le couple créateur / créature s'est-il défait et le vieux grand-père reste-t-il seul et privé d'avenir, tandis que Pascal-Pygmalion se prolonge en Clotilde, mais aussi en l'enfant qui naît d'elle, ayant réussi à conjuguer, fait rare dans l'imaginaire dix-neuviémiste, œuvre d'art (ou de savoir) et œuvre de chair.

\section{Deux testaments littéraires?}

Le mythe de Pygmalion est aussi pertinent pour la lecture conjointe des deux romans, en ce que le personnage masculin semble, sur bien des points, une transposition du romancier, et une figure de créateur d'autant plus explicite. Chacun des deux romans a par ailleurs une dimension éminemment réflexive, en ce qu'il s'interroge sur l'esthétique mise en œuvre, pour les Goncourt, dans toute leur production romanesque, pour Zola, dans l'ensemble du cycle des Rougon-Macquart arrivé à son terme.

Les données autobiographiques présentes dans Le Docteur Pascal sont connues : Zola projette sur son personnage son amour pour Jeanne Rozerot, devenue en décembre 1888 sa maîtresse, et qui lui donne une fille en 1889 et un fils en 1891, alors que son union avec Alexandrine Zola reste stérile. Zola partage aussi avec son personnage de savant les outils qui lui ont servi à bâtir son cycle romanesque : son arbre généalogique, la théorie de l'hérédité qui l'informe et la méthode documentaire qui l'étaie, et que le romancier a pour sa part mise en pratique bien au delà du seul domaine médical. Mais, comme l'a analysé J.-L. Cabanès, dans ce dernier roman qui vient clore un cycle, l'adéquation entre le

1. Chérie, op. cit., chap. LXXVIII, p. 242.

2. Ibid., chap. LXXVIII, p. 243. On est proche aussi de l'auto-érotisme qu'on observe plus loin avec le parfum (autre composante de la parure), et présent déjà chez Manette Salomon ou chez Nana. 
romancier et son personnage, comme le retour réflexif sur les Rougon-Macquart, sont poussés encore plus avant :

En résumant avec un art consommé et varié les autres romans de la série, tout en faisant en sorte que Pascal mette en récit l'arbre généalogique des Rougon-Macquart, l'écrivain raconte en effet ses propres œuvres, tantôt en les regroupant par ensembles de familles, tantôt en dégageant de manière synthétique les schèmes symboliques qui leur servent de principes organisateurs. Il propose ainsi un parcours de lecture des œuvres qu'il a déjà publiées. Il donne, à leur éventuel lecteur, une sorte de mode d'emploi. À cela vient s'ajouter un autre jeu de miroir. Si l'écrivain fonde ses romans sur des dossiers préparatoires, le docteur Pascal lit à sa nièce des dossiers qui sont en fait le sommaire ou le sublimé des Rougon-Macquart ${ }^{1}$.

C'est ainsi à la fois la genèse du texte et sa réception qui sont mises en abyme dans l'ultime roman de Zola, par une mise en scène complexe des actes de lecture et d'écriture, dont le plus troublant est sans doute celui où Pascal mourant inscrit lui-même sur l'arbre généalogique la date de sa mort : c'est le geste scriptural du romancier décidant du destin du personnage qui est ainsi donné à voir dans la fiction, en un effet de signature particulièrement voyant, puisqu'il s'accompagne d'une datation (sur le symbolisme de laquelle il conviendrait sans doute de s'interroger) : "Meurt d'une maladie de cœur, le 7 novembre 1873 ». Cet effet de signature est souligné par plusieurs dispositifs : par sa position en fin de chapitre, par l'isolexisme qui vient clore définitivement celui-ci ( $P$ Pascal mourut. [...] Et ce fut la mort, très simple.»), enfin par une stylistique de la brevitas, dans ces phrases doublement conclusives, puisqu'elles scellent la fin du cycle et du personnage qui en est son principe ordonnateur : elles viennent dire la cessation de la vie, devant le fait brut de la mort, mais aussi la fin de la parole et de l'écriture, qui se referme sur un unique nom propre.

Cet effet de signature particulièrement voyant, on peut légitimement se demander s'il n'a pas été inspiré par Chérie. L'intrigue de Chérie, comme celle du Docteur Pascal, transpose des données biographiques qui conferent une place dans le texte au romancier et aux femmes qu'il a aimées : à travers la geste militaire de la lignée Haudancourt, c'est la propre ascendance d'Edmond qui se trouve convoquée ; et Chérie mêle divers emprunts, à Marie Abbatucci, à Pauline Zeller, qui fréquentent le salon de la princesse Mathilde dans les années 1870-1880, ou à ces souvenirs plus lointains de Blanche Passy, ou les petites Marcille. C'est ainsi un itinéraire affectif dont le roman se fait discrètement l'écrin, en même temps qu'il convoque, par diverses allusions, les autres hérö̈nes malheureuses des Goncourt (Germinie cachant sa mort dans une fascination des amours d'autrui, Philomène transparaissant dans la spectatrice qui

1. Introduction au Docteur Pascal, op. cit., p. 32. 
attend le bistouri du chirurgien, Renée à laquelle ses parents vont survivre, Élisa abîmée dans le regret de son enfance vosgienne...). Cette lecture autobiographique et réflexive, la préface de Chérie y invite quant à elle explicitement : après un positionnement théorique assez ferme, sur sa méthode documentaire, sur l'avenir du roman, sur la question du style, Edmond de Goncourt conclut en désignant sa préface comme un " testament littéraire " qui signe ses adieux au genre romanesque, au profit de son Journal auquel il va désormais consacrer ses dernières années. Offrant alors en guise de prémices la préface de ce Journal, il fait revivre le travail conjoint avec son frère mort, auquel il donne finalement directement la parole, dans le bilan littéraire que celui-ci avait semblé dresser peu avant de mourir, sur « la recherche du vrai en littérature, la résurrection de l'art du XVIII siècle, la victoire du japonisme " ${ }^{1}$ opérées selon lui par leurs soins. Cette mise en scène du "promeneur mourant de l'allée du Bois de Boulogne " à la toute fin de la préface, entre en résonance directe avec la fin du roman, puisque, comme l'a souligné l'édition de Jean-Louis Cabanès et Philippe Hamon, le fac-similé du faire-part de décès de l'héroöne porte la date précise du décès de Jules de Goncourt. Dans cet ensemble textuel complexe (roman et préface ou plutôt double préface) fortement marqué par une démarche conclusive mais aussi inaugurale (Goncourt amorce un travail de relecture du Journal, lequel ne doit paraître que "vingt ans après [sa] $\left.\operatorname{mort}^{3} »\right)$, Zola a sans nul doute été marqué par les multiples effets de signature par lesquels le texte désigne les étapes de sa genèse (dans ses entrelacs avec la vie affective de son auteur) et prépare les conditions de sa réception, tout en fonctionnant, sur le plan personnel, sur le mode de la relique, ce dont atteste, chez Zola, et la double dédicace (officielle, à sa mère et à sa femme, manuscrite, à Jeanne Rozerot sur l'exemplaire qu'il lui offre), et le terme même de relique désignant l'Arbre manuscrit échappé aux flammes et les derniers mots au crayon de la main de Pascal ${ }^{4}$.

\section{Le décor et la parure}

On peut également approfondir l'analogie entre les deux romans en examinant, après la dimension réflexive et la proximité entre le personnage masculin et le romancier, le traitement du personnage féminin et la façon dont il convoque une très riche intertextualité sur la question du décor.

Dans le dernier tiers du siècle, où fleurissent publications de modes (Mallarmé, Uzanne...) et manuels de décoration intérieure (Charles Blanc, Henry Havard...), comment donner le premier rôle à un personnage féminin sans évoquer sa demeure ? Il y a de fait un vaste ensemble de textes narratifs ou poétiques comportant des descriptions d'intérieurs que retravaille Zola (le «Frisson

1. Chérie, op. cit., Préface, p. 53.

2. Ibid.

3. Ibid., Préface, p. 17.

4. Le Docteur Pascal, op. cit., chap. XIV, p. 505. 
d'hiver " de Mallarmé, la "Véra " de Villiers'), et Chérie, qui évoque certes davantage les parures que les décors, doit certainement être considéré comme l'un d'eux. Loin d'avoir une finalité seulement esthétique, ces textes, d'une part, opèrent un glissement de l'intimité spatiale à l'intimité psychologique et questionnent tant l'identité individuelle que la validité du couple (et nous pourrons alors approfondir les analyses esquissées en première partie sur la solitude ou la complémentarité des personnages); ces écrits, d'autre part, parce qu'ils mettent en scène un décor toujours hérité, posent la question de la gestion du passé, et de son ouverture à l'avenir. Associé au motif de la filiation (lui-même lié à l'existence et au fonctionnement du couple), le décor permet, dans les textes littéraires de la fin du siècle, de penser l'inscription dans l'histoire et la possibilité d'un devenir.

Le décor de la chambre de Chérie, moins décrit que les robes que la jeune fille porte tout au long du roman, scande cependant les deux temps forts du roman: l'apothéose de son génie créateur, l'agonie dans laquelle son corps semble se venger. Dans les deux cas, dans son exclusive blancheur, ce décor est le symbole transparent d'une virginité d'abord triomphante puis douloureusement subie. Il est surtout intéressant par la référence historique qu'il propose : c'est un mobilier ancien, mais paradoxalement converti d'emblée en marqueur de la jeunesse de l'héroïne :

Le mobilier de la chambre, un ancien mobilier du Premier Empire, est tout à fait jeune fille. Ce sont des fauteuils, des chaises en acajou, avec des coussins volants en piqué blanc, où l'on voit, brodés très en relief et également en blanc, deux cols de cygne accolés au dessus d'un grand chiffre, qui par une coïncidence bizarre se trouve être un $C^{2}$.

Chez Zola, la chambre de Clotilde est elle aussi de style Empire, mais témoigne d'ancrages historiques plus anciens, et plus éclectiques :

Bien que la Souléiade datât du siècle dernier, on avait dû la remeubler sous le Premier Empire, car il y avait là, pour tenture, une ancienne indienne imprimée, représentant des bustes de sphinx, dans des enroulements de couronne de chêne. Autrefois d'un rouge vif, cette indienne était devenue rose, d'un vague rose qui tournait à l'orange. [...] Et c'était vraiment exquis cette pourpre effacée, ce ton d'aurore si délicatement doux. Quant au lit [...] il tombait d'une vétusté telle qu'on avait dû le remplacer par [...] un autre lit Empire, bas et très large, en aca-

1. Mallarmé, « Frisson d'hiver ", composé en 1864, paru en 1867, repris dans Divagations (1897), Villiers de l'Isle-Adam, Véra (1874) repris dans Contes cruels (1883).

2. Chérie, op. cit., chap. LXII, p. 206. E. de Goncourt prend acte du retour en grâce du style Empire à la fin du XIX siècle, alors qu'il l'a sévèrement critiqué au temps où il était un thuriféraire du rococo (voir mon article "Mme Récamier et la décoration d'intérieur : évolution d'un réseau d'images (XIX'-XXe siècles)", p. 185-211 dans Juliette Récamier dans les arts et la littérature. La fabrique des représentations, Delphine Gleizes et Sarga Moussa (dir.), Hermann, 2011). 
jou massif, dont les quatre colonnes d'angle portaient aussi des bustes de sphinx, pareils à ceux de la tenture. D'ailleurs, le reste du mobilier était appareillé, une armoire à portes pleines et à colonnes, une commode à marbre blanc cerclé d'une galerie, une haute psyché monumentale, une chaise longue aux pieds raidis, des sièges aux dossiers droits, en forme de lyre. Mais un couvre-pied, fait d'une ancienne jupe de soie Louis XV, égayait le lit majestueux ${ }^{1}[\ldots]$

Zola transpose ici la formule essentielle de "Frisson d'hiver ", «la grâce des choses fanées ", et insiste sur l'usé, symbole, comme chez Mallarmé, non tant de décrépitude que de douceur, et ici d'emblée associé à la possibilité d'une renaissance, comme le suggère le sémantisme du terme d'aurore. En outre, l'éclectisme du décor suggère une sédimentation de différentes strates temporelles, fondues dans une même persistance, et sur lesquelles le présent et le futur prendront mieux racine, comme cela apparait à la fin du roman ${ }^{2}$.

Si chez Goncourt et Zola le décor semble d'abord traduire une gestion positive des héritages, où s'enracine le renouveau de l'enfance ou de la jeunesse, chez Goncourt il demeure le cadre d'une solitude stérile; chez Zola, qui exacerbe quant à lui la tension entre l'ancien et le nouveau, le décor évolue au contraire vers la représentation d'un cocon qu'on partage, non seulement parce qu'il devient le lieu d'une étreinte amoureuse féconde, mais aussi parce qu'il construit une réciprocité dans l'expérience affective du manque, réciprocité qui permettra à l'attachement conjugal de persister par delà la mort. Comme le comte d'Athol, époux de Véra dans la nouvelle de Villiers, Pascal d'abord éprouve dans la chambre de Clotilde la douleur de son absence par le biais des marques qu'elle a laissées :

Il en gardait la clef, il n'y avait pas dérangé un objet de place depuis le triste matin du départ ; et une jupe oubliée traînait encore sur un fauteuil. Là, il respirait jusqu'à son souffle, sa fraîche odeur de jeunesse, restée parmi l'air comme un parfum. Il ouvrait ses bras éperdus, il les serrait sur son fantôme, flottant dans le tendre demi-jour des volets fermés, dans le rose éteint de la vieille indienne des murs, couleur d'aurore. Il sanglotait devant les meubles, il baisait le lit, la place marquée où se dessinait l'élancement divin de son corps ${ }^{3}$.

Mais, Pascal mort, c'est Clotilde qui cherche dans le même lieu l'illusion de sa présence. Et la permanence des choses ne dit plus seulement l'absence mais permet bien l'enracinement d'une nouvelle existence :

1. Le Docteur Pascal, op. cit., chap. II, p. 76.

2. Ibid., chap. XIV, p. 492. Par ailleurs Clotilde ne veut pas changer de chambre, au moment où Pascal est pris d'une frénésie du don pour la parer, et semble ainsi manifester son attachement à la continuité d'un héritage (ibid., chap. VIII, p. 287).

3. Ibid., chap. 12, p. 421-422. 
Il lui semblait, par moments, que Pascal y vivait encore, car elle n'y avait rien dérangé de leur existence de jadis. Les meubles étaient aux mêmes places [...]. C'était toujours la même chambre douce, aux antiques meubles familiers, aux tentures attendries par l'âge, couleur d'aurore, la très vieille chambre que l'enfant rajeunissait de nouveau ${ }^{1}$.

On pourrait mener une semblable analyse à partir d'un modeste élément de parure comme le collier de perles, qui apparaît dans les deux romans à des moments clé de l'intrigue. Certes, chez Goncourt, il résume une série de dons du grand-père, comme Chérie le rappelle; mais elle ne voit plus à travers lui que le bijou préféré ( Des perles, moi, je trouve cela décidément plus joli que les diamants $\left.{ }^{2} ! »\right)$. Chez Zola au contraire, le don des perles, dramatisé (Pascal, à cours d'argent, est en proie à une lutte intérieure) suscite un contre-don symbolique, et amène l'étreinte amoureuse :

D’une étreinte il la saisit, il la mena devant la grande psyché où elle se vit toute. A son cou, la mince chaîne n'était qu'un fil d'or, et elle aperçut les sept perles comme des étoiles laiteuses, nées là et doucement luisantes sur la soie de sa peau. [...] Et la joie qu'elle avait dans les yeux, cette joie de femme et d'amante, ravie d'être belle, d'être adorée, le récompensait divinement de sa folie".

Le collier demeure par ailleurs, comme la chambre, un objet intime qui atteste de la permanence du couple, puisque, Pascal mort, il n'apparait au cou de la jeune femme que lorsque celle-ci met son enfant au sein ${ }^{4}$.

On a vu en outre ici que la psyché, autre objet auquel les deux textes se réferent, donne lieu chez Zola à une contemplation commune, alors que Chérie se complait dans un reflet solitaires. Narcisse supplante Galatée chez Goncourt, alors qu'un autre modèle, d'inspiration biblique (le roi David et la jeune Abisaïg), est récurrent chez Zola, pour dire l'alliance persistante et féconde de la vieillesse et de la jeunesse, et la façon dont le couple s'inscrit fondamentalement dans la transmission d'un héritage.

\section{La collection : triomphe ou liquidation ?}

Le décor, on l'a vu, pose la question de l'héritage et de sa transmission. Il rencontre souvent, dans les romans de la fin-de-siècle, une autre thématique,

1. Ibid., chap. 14, p. 492.

2. Chérie, op. cit, chap. CIII, p. 297.

3. Le Docteur Pascal, op. cit., chap. X, p. 351-352.

4. Ibid., chap. XIV, p. 508.

5. Chérie, op. cit., chap. LXXVIII, p. 242. 
celle de la collection, dont il est le versant moins obsessionnel et plus familier ${ }^{1}$. Or la collection, tant comme référent que comme élément structurant de l'écriture romanesque, est effectivement un autre point de rencontre, et de divergence, des deux textes en question, Chérie semblant célébrer le triomphe de la collection, alors que Le Docteur Pascal procède à l'inverse à sa liquidation, et formule ainsi, plus résolument que par le symbolisme du décor, une idéologie dynamique du devenir.

Le projet du roman de Goncourt s'est formulé, dès l'origine, à partir d'un objet emblématique contemplé dans une vitrine, comme "une collection de toutes les élégances matérielles, morales, sentimentales du jour d'aujourd'hui ${ }^{2}$ ». La collection apparaît ainsi comme un référent essentiel (le roman se présente à bien des égards comme une collection de mode), et comme un modèle structurant (dont la définition du roman comme " collection de documents humains" dans la préface des Frères Zemganno avait en 1879 théorisé le principe). La nouvelle stratégie documentaire qu'Edmond de Goncourt a mise en place, en octobre 1881, dans la Préface à la première édition de La Faustin, où, au lieu de présenter son nouveau roman, il a exposé le projet du suivant, consolide encore ce principe :

Aujourd'hui, lorsqu'un historien se prépare à écrire un livre sur une femme du passé, il fait appel à tous les détenteurs de l'intime de la vie de cette femme, à tous les possesseurs de petits morceaux de papier, où se trouve raconté un peu de l'histoire de l'âme de la morte.

Pourquoi, à l'heure actuelle, un romancier (qui n'est au fond qu'un historien des gens qui n'ont pas d'histoire), pourquoi ne se servirait-il pas de cette méthode, en ne recourant plus à d'incomplets fragments de lettres et de journaux mais en s'adressant à des souvenirs vivants, peut-être tout prêts à venir à lui ${ }^{3}$ ?

Certes, cet appel à documents n'a pas tenu toutes ses promesses, mais le texte de Chérie reste essentiellement composé d'une multitude de documents variés, qui sont juxtaposés, et non fondus, pour ne pas entraver ce pouvoir de dévoilement dont ils sont le siège, boîtes symboliques qu'il faut apprendre à ouvrir pour voir s'en dégager le sens. Celui-ci ne naît plus du dynamisme de l'intrigue, de son nœud, de sa résolution, mais d'un montage complexe de ces objets rares et précieux que sont les "documents intimes". D'où parfois un soulignement voyant de leurs contours : l'étroit cadrage du chapitre autour d'un seul type de document (la liste des bals, chap. LXVI ; la lettre de la jeune ma-

1. Voir la troisième partie de mon essai Poétique de la collection au XIX siècle. Du document de l'historien au bibelot de l'esthète. Nanterre, Presses universitaires de Paris-Ouest (Orbis litterarum), 2010.

2. Journal, 2 octobre 1864, éd. J.-L. Cabanès, Paris, Champion, 2013, t. III-1, p. 795.

3. Préface de la première édition de La Faustin, octobre 1881, dans Préfaces et manifestes littéraires (1888), Paris, Flammarion et Fasquelle, [1926], p. 57. 
riée, chap. XCIII) ; les points de suspension lorsqu'un document trop long est tronqué (par exemple par le Règlement de vie, chap. XXXIII) ; le recours à une typographie spécifique (le rébus du Maréchal, chap. XLIX ; le faire-part de décès, chap. $\mathrm{CV}$ ). Tout cela rend l'insert plus marqué, et relève d'une esthétique du montage qu'avait bien mise en lumière l'édition de P. Hamon et J.-L. Cabanès.

Le dernier mot reste enfin à cet étrange fac-similé du faire-part de décès, qui semble un document authentique, jusque dans le nom de l'imprimeur, mais qui, par sa fidèle transposition d'un usage commercial du langage (juxtapositions et abréviations dans les noms propres de lieu et de personnes par lesquels s'identifie l'imprimeur: "Bar-le-duc, Imp. Veuve Numa Rolin Choquet et Cie ») suggère aussi la menace que l'excès documentaire fait peser sur le roman.

Dans Le Docteur Pascal, le modèle de la collection documentaire est bien évidemment visualisé par les dossiers de Pascal, classés, constitués en série, et qui proposent une structure d'ensemble au sein de laquelle l'hétérogénéité de chaque pièce prend sens ${ }^{1}$ :

Sur cette planche élevée, toute une série d'énormes dossiers s'alignaient en bon ordre, classés méthodiquement. C'étaient des documents divers, feuilles manuscrites, pièces sur papier timbré, articles de journaux découpés, réunis dans des chemises de fort papier bleu, qui chacune portait un nom écrit en gros caractères. On sentait ces documents tenus à jour avec tendresse, repris sans cesse et remis soigneusement en place; car, de toute l'armoire, ce coin-là seulement était en ordre ${ }^{2}$.

L'arbre généalogique, motif structurant de cette collection documentaire, la rapproche aussi du modèle encyclopédique (qu'on songe aux arborescences qui ouvrent l'Encyclopédie de Diderot et d'Alembert). On a donc bien affaire ici à une collection à visée scientifique; Pascal met en œuvre une démarche tout à la fois de collectionneur et de savant: la diversité matérielle des supports documentaires, qui témoigne de leur diversité d'origine, fait aussi la richesse de l'ensemble qui les subsume et atteste du travail de collecte et d'interprétation du savant.

Mais finalement, alors que le décor inchangé permet l'enracinement du nouveau dans l'ancien, les documents sont congédiés, seul est préservé le dispositif structurant de l'arbre généalogique, converti de principe de classement en principe de vie. Le soigneux compartimentage de la collection se défait ainsi, d'une part sous l'effet du feu qui consume les dossiers, d'autre part sous l'effet de l'élan vital, qui parcourt toutes les branches de l'arbre et les unifie dans une

1. Cependant le terme de collection n'apparaît pas, et Zola prend également soin de l'éviter dans la plupart de ses écrits théoriques, pour se démarquer d'Edmond de Goncourt (la formule de "collection de documents humains " de la préface des Frères Zemganno a cristallisé la polémique entre les deux écrivains autour de 1878-1879).

2. Le Docteur Pascal, op. cit., chap. I, p. 45. 
même poussée, dans la lecture que Clotilde en fait à la fin du roman ${ }^{1}$. De la même façon, les plantes du Paradou débordent les bordures jadis dessinées, et Mouret bouscule in extremis le classement du grand magasin, pour qu'il soit mieux parcouru par le flux désorienté de la clientèle. On peut donc se demander si la collection et son étiquetage ne sont là chez Zola que pour être dépassés, au profit d'une logique non plus savante mais organique et vitale.

Combler les manques, rétablir le continu, tendre vers une dynamique du flux, est un principe théorique qu'il a souvent affirmé, contre Edmond de Goncourt qui semble vouloir en rester à la compilation dans la préface des Frères Zemganno ("immenses emmagasinements d'observations", "innombrables notes $\left.{ }^{2} » ..\right)$ :

Prenez au contraire des faits vrais que vous avez observés autour de vous, classez-les d'après un ordre logique, comblez les trous par l'intuition, obtenez ce merveilleux résultat de donner la vie à des documents humains, une vie propre et complète, adaptée à un milieu, et vous aurez exercé dans un ordre supérieur vos facultés d'imaginer. Eh bien! notre roman naturaliste est justement le produit de ce classement des notes et de l'intuition qui les complète ${ }^{3}$.

En soulignant la part de "l'intuition qui complète ", Zola montre comment la faculté créatrice du romancier s'exerce pour restituer la vie " complète ".

Dans Le Docteur Pascal, succédant à l'image du feu qui consume, celle de la sève liquide qui coule au cœur de l'arbre impose un imaginaire de la poussée vitale, de la continuité qui lie une collectivité, à l'opposé du morcellement, du discontinu, du solitaire et du figé chez Goncourt. On pourrait donc conclure, de Chérie au Docteur Pascal, à un passage de relais entre deux modèles épistémologiques divergents, l'un taxinomique et statique, l'autre dynamique et énergétique, passage de relais grâce auquel les héritages de tous ordres (biologiques, historiques et culturels) vont commencer à être pensés sur le mode, non plus du permanent, mais du dépassement, voire du reniement, de la table rase où pourra prendre racine un avenir qu'on souhaite riche de tous les possibles.

Dominique PETY

1. Ibid., chap. XIV, p. 506.

2. Voir Préface des Frères Zemganno, Euvres Complètes, t. IX, éd. de Catherine DousteyssierKhose, Paris, Champion, 2012, p. 112.

3. "Les documents humains" (1879), Le Roman expérimental, CEuvres Complètes, t. X, Paris, éd. Tchou, 1968, p.1317. 


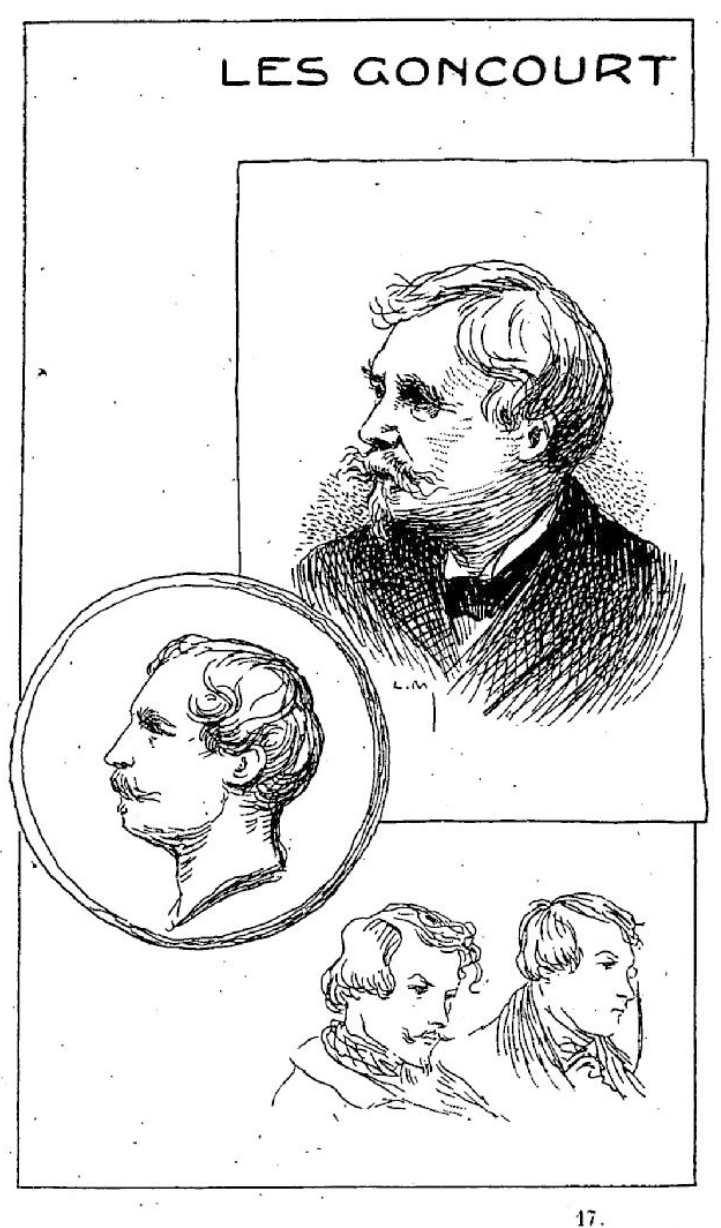

1. Lucien Métivet, "Les Goncourt", in Édouard Drumont, Les Tréteaux du succès. Figures de bronze ou statues de neige, [Paris], Flammarion, 1900. 


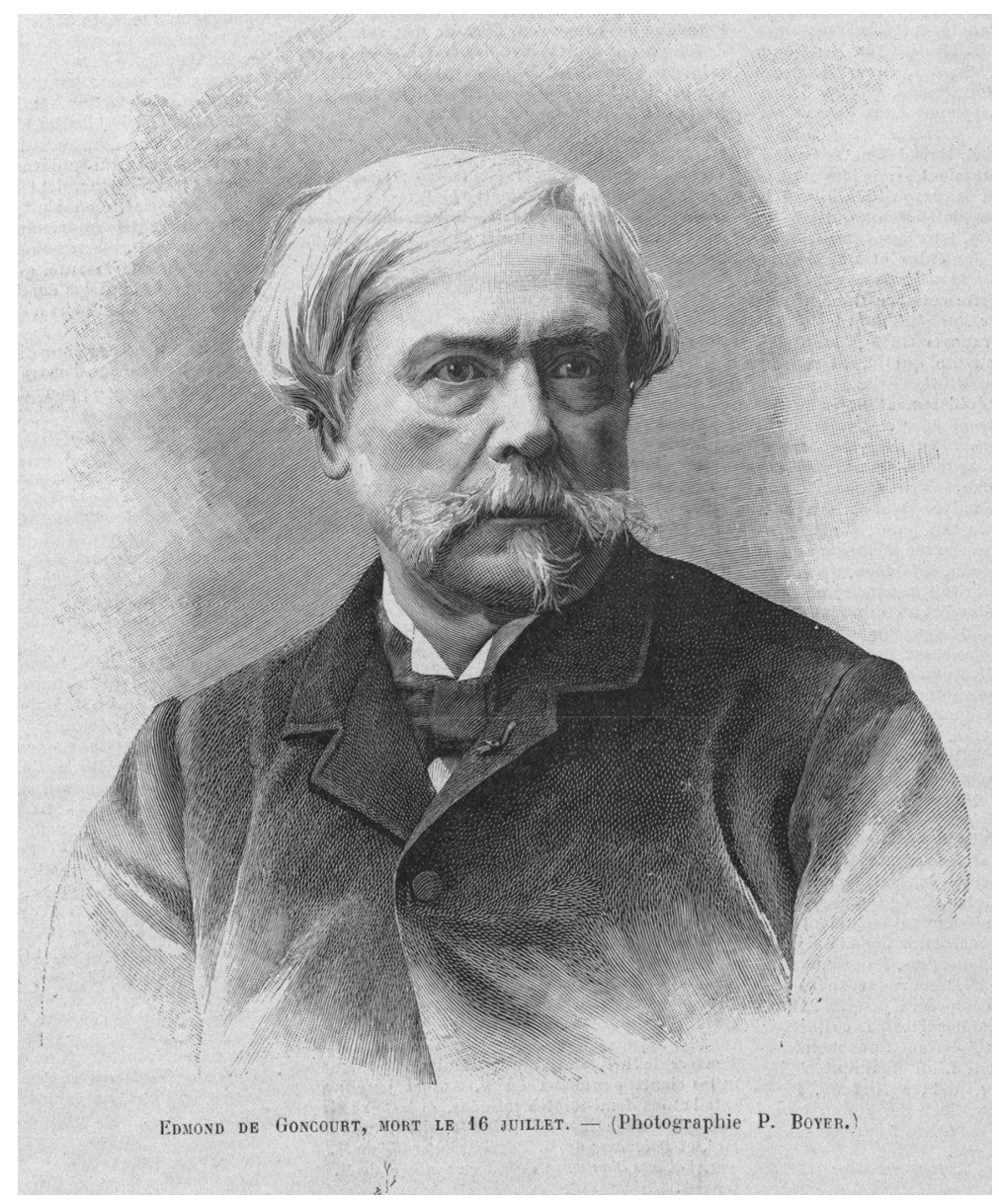

2. P. Boyer, Photographie d'Edmond de Goncourt, parue dans Le Monde illustré, 25 juillet 1896, T. 79 , p. 53 («Edmond de Goncourt », nécrologie anonyme). 


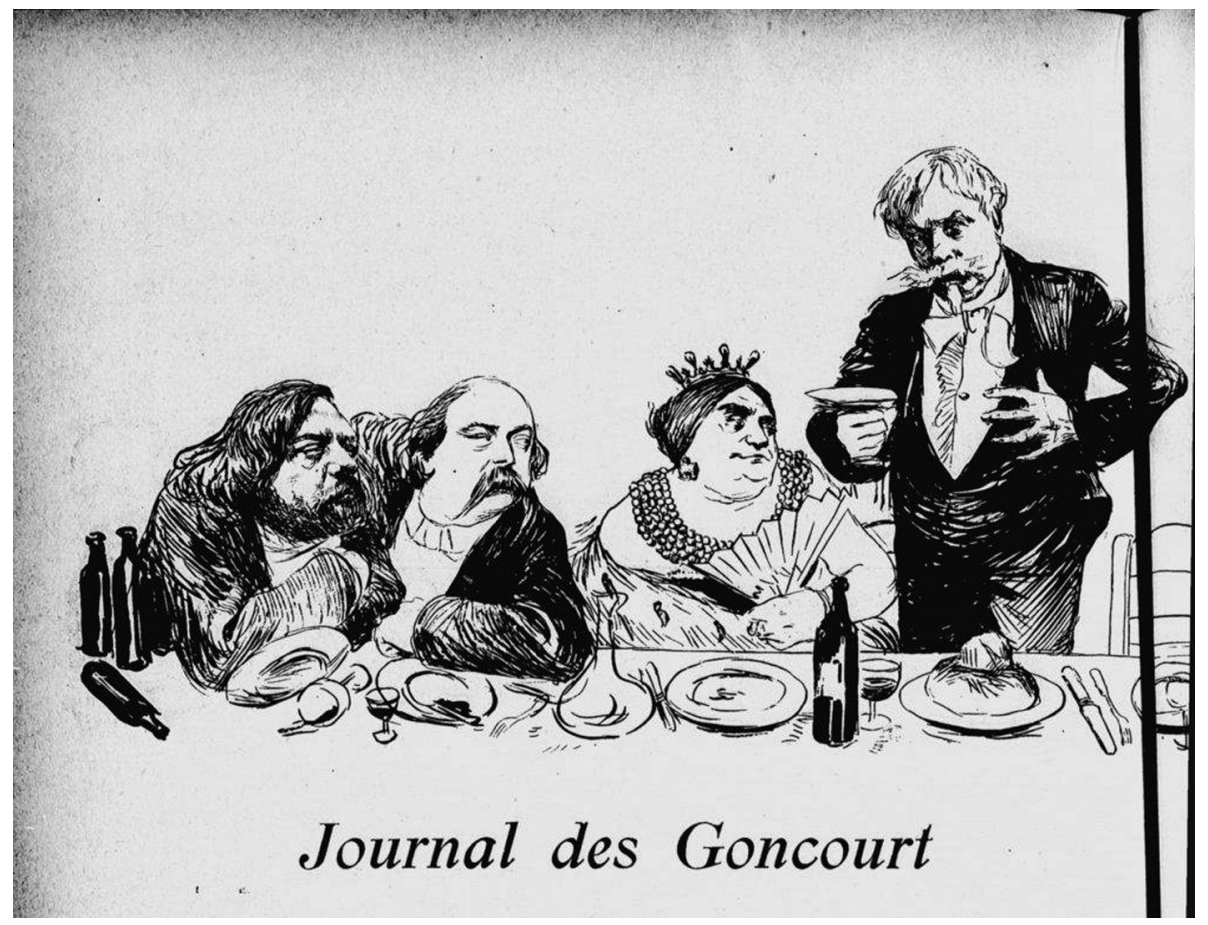

3. Jean Veber, illustration du chapitre "Journal des Goncourt", in Pierre Veber, Les Veber's. Les Veber's. Les Veber's [Texte satirique de Pierre Veber, illustré de 350 dessins par Jean Veber], Paris, E. Testard, 1985, p. 168. 


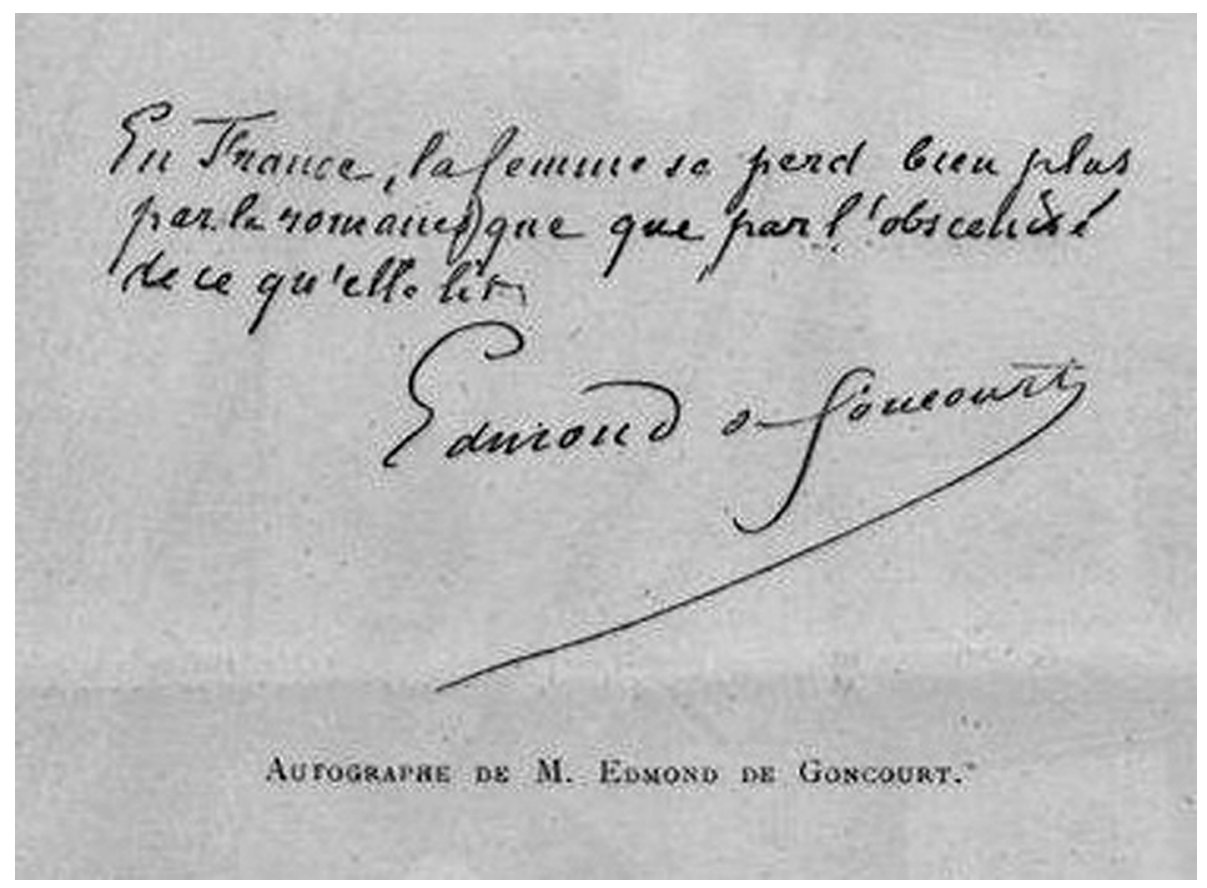

4. Autographe d'Edmond de Goncourt reproduit dans l'article de G. Lenôtre, « Nos contemporains chez eux. M. Edmond de Goncourt ", Le Monde illustré, 21 février 1891, T. 68, p. 154 


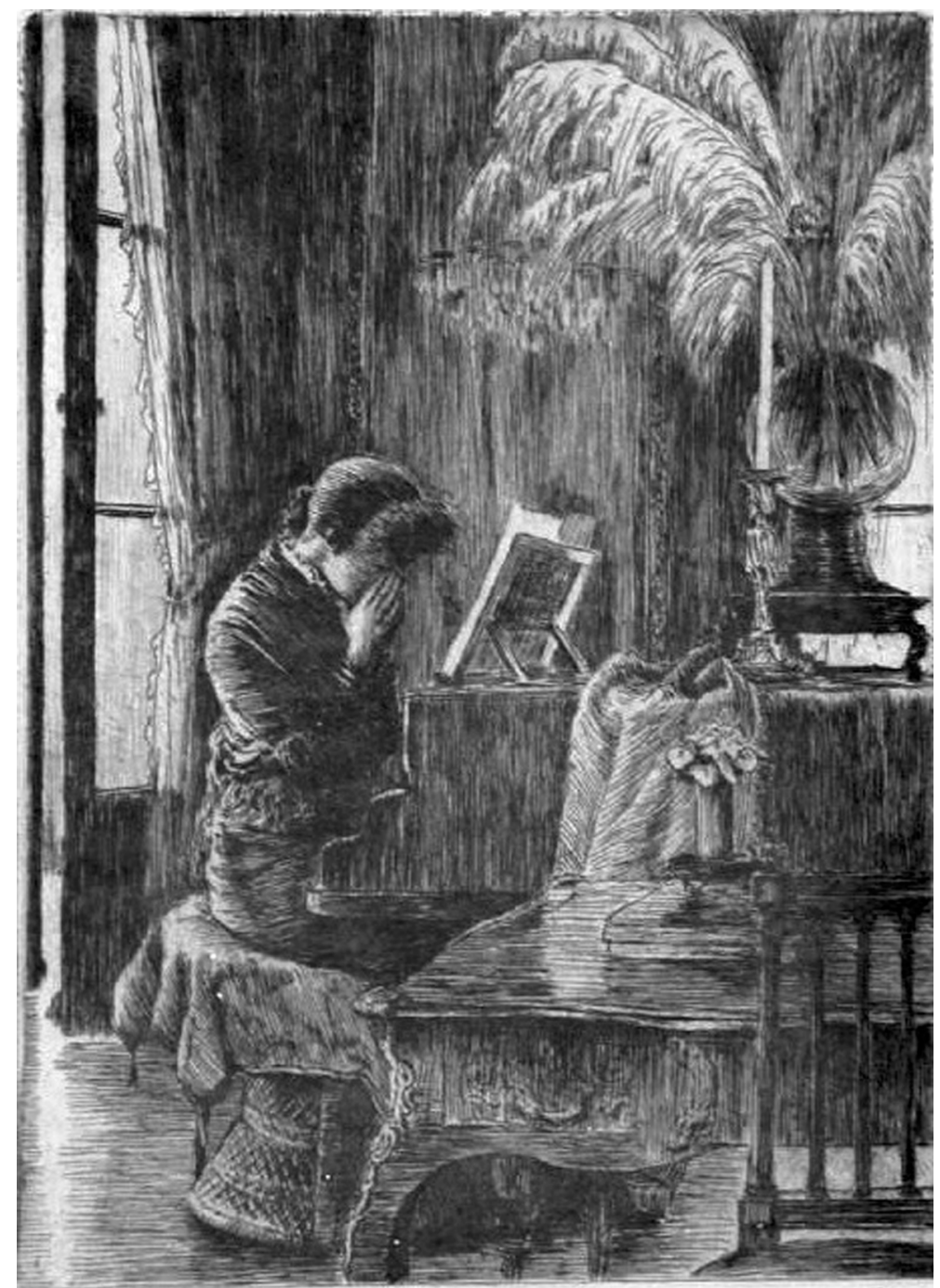

5. James Tissot, Renée Mauperin pleurant devant son piano, eau-forte, $14 / 9,8 \mathrm{~cm}$, illustration de Renée Mauperin, Paris, Charpentier, 1884 


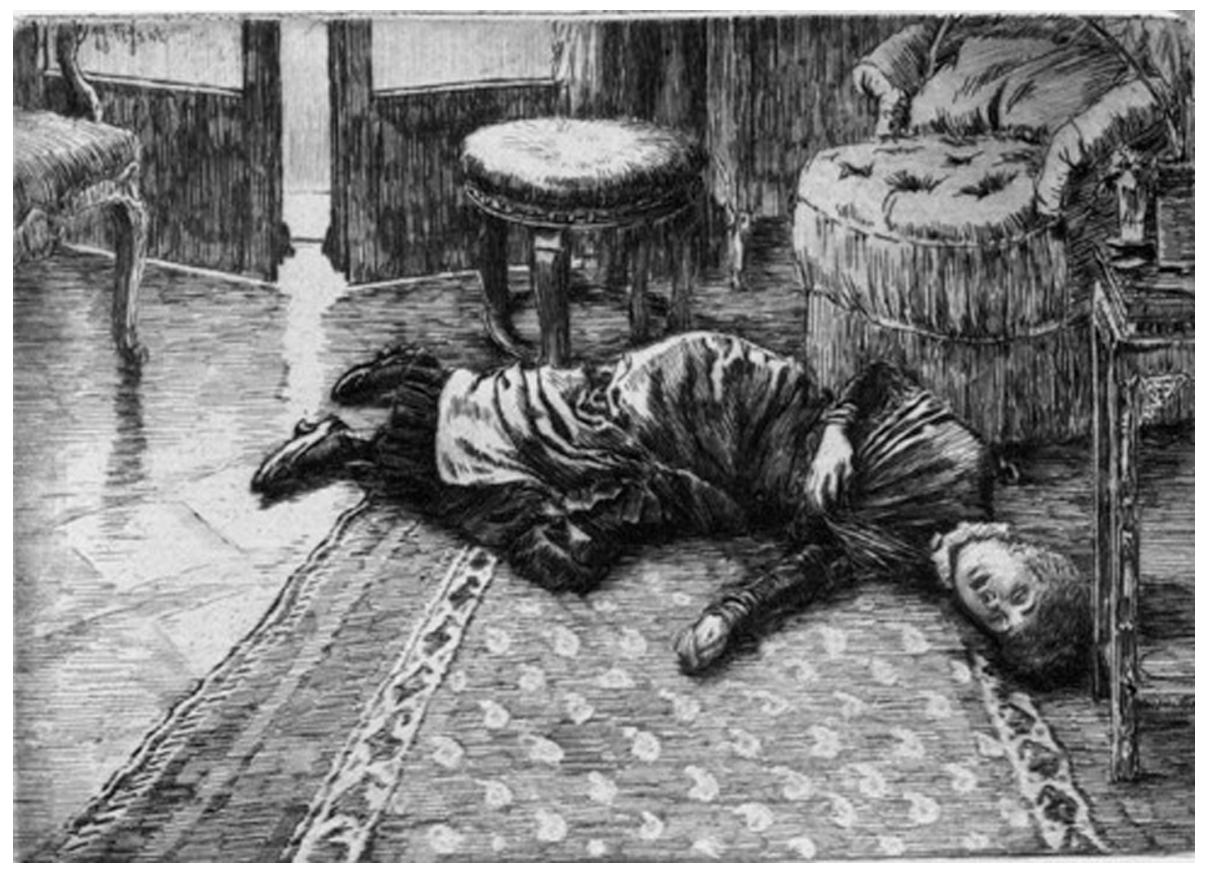

6. James Tissot, Renée après avoir appris la mort de son frère, $14 / 9,8 \mathrm{~cm}$, illustration de Renée Mauperin, Paris, Charpentier, 1884 